OPEN ACCESS

Edited by:

Matthew A. Wyon,

University of Wolverhampton, UK

Reviewed by:

Derrick D. Brown

Donders Institute for Brain, Cognition and Behaviour, Netherlands

Christopher Fullerton,

University of Wolverhampton, UK

*Correspondence: Ralf Brand

ralf.brand@uni-potsdam.de

Specialty section:

This article was submitted to

Performance Science,

a section of the journal

Frontiers in Psychology

Received: 02 October 2015 Accepted: 19 January 2016

Published: 09 February 2016

Citation:

Brand $R$ and Koch H (2016) Using

Caffeine Pills for Performance

Enhancement. An Experimental Study on University Students' Willingness and Their Intention to Try

Neuroenhancements.

Front. Psychol. 7:101.

doi: 10.3389/fpsyg.2016.00101

\section{Using Caffeine Pills for Performance Enhancement. An Experimental Study on University Students' Willingness and Their Intention to Try Neuroenhancements}

\author{
Ralf Brand* and Helen Koch \\ Sport and Exercise Psychology, University of Potsdam, Potsdam, Germany
}

Recent research has indicated that university students sometimes use caffeine pills for neuroenhancement (NE; non-medical use of psychoactive substances or technology to produce a subjective enhancement in psychological functioning and experience), especially during exam preparation. In our factorial survey experiment, we manipulated the evidence participants were given about the prevalence of NE amongst peers and measured the resulting effects on the psychological predictors included in the PrototypeWillingness Model of risk behavior. Two hundred and thirty-one university students were randomized to a high prevalence condition (read faked research results overstating usage of caffeine pills amongst peers by a factor of 5; 50\%), low prevalence condition (half the estimated prevalence; 5\%) or control condition (no information about peer prevalence). Structural equation modeling confirmed that our participants' willingness and intention to use caffeine pills in the next exam period could be explained by their past use of neuroenhancers, attitude to NE and subjective norm about use of caffeine pills whilst image of the typical user was a much less important factor. Provision of inaccurate information about prevalence reduced the predictive power of attitude with respect to willingness by $40-45 \%$. This may be because receiving information about peer prevalence which does not fit with their perception of the social norm causes people to question their attitude. Prevalence information might exert a deterrent effect on NE via the attitude-willingness association. We argue that research into NE and deterrence of associated risk behaviors should be informed by psychological theory.

Keywords: attitude, prevalence information, prototype-willingness-model, social reactivity, doping

\section{INTRODUCTION}

Neuroenhancement (NE) is non-medical use of psychoactive substances or technology for the purpose of producing a subjective enhancement in psychological functioning and experience (Jongh et al., 2008; Mueller and Schumann, 2011; de Berker et al., 2013; Kipke, 2013; Clark and Parasuraman, 2014; Farah et al., 2014; Maier and Schaub, 2015, for elements of this definition). Sometimes the term 'pharmacological cognitive enhancement' is used to refer to the use of psychoactive drugs to enhance psychological capacities such as attention, concentration, and 
memory (e.g., Franke and Lieb, 2010; Dietz et al., 2013). The performance-enhancing effects of drugs used as neuroenhancers seem to be limited, regardless of whether they are prescription drugs such as methylphenidate (Ritalin), mixed amphetamine salts (Adderall) and modafinil (Provigil); illicit drugs (e.g., amphetamine, cocaine, ecstasy) or over-the-counter products (e.g., caffeinated drinks, energy drinks, Ginkgo biloba; e.g., Franke et al., 2014). Given the considerable overlap between the inconsistent cognitive effects of most of these substances on cognitive capacity and their incidental mood- and motivationrelated effects (e.g., Ilieva et al., 2013) describing them as 'cognitive enhancers' may be overly narrow and restrictive (Zohny, 2015).

Wolff and Brand (2013) pointed out that information about the type and effects of a substance does not explain why people do or do not experiment with neuroenhancers. They argued that motivation to experiment with neuroenhancers could be explained by expectations about their effects. Someone drinking a strong cup of coffee explicitly in order to stay awake and thus study for longer or to enhance specific cognitive capacities (e.g., attention, recall memory) thus provides an adequate example of attempted NE. In this article, we will treat NE as goal-directed behavior intended to produce an improvement in academic performance.

There is an active debate about the ethics and pros and cons of NE at present and in the future (e.g., Hildt and Franke, 2013). Scientists remind us not to overestimate the effects of substances used as neuroenhancers (e.g., Franke et al., 2014; Zohny, 2015), but the market for substances (e.g., soft-drinks) promising an energy boost, or cognitive benefits as well as mood enhancement (Ishak et al., 2012) is increasing. In the light of these developments it is especially important to avoid fuelling the media hype surrounding NE (Partridge et al., 2011) and to state clearly the aims and limitations of published research. This paper is on the psychology of NE. We intend to describe and analyze university students' motivation to experiment with $\mathrm{NE}$ in terms of a social cognitive theory of behavior.

\section{Prevalence of NE in Academia}

There is limited reliable evidence on prevalence of NE in academia. Extant studies differ with regard to underlying definitions (e.g., whether NE is restricted to use of prescription drugs), representativeness of the sample (ad hoc; random), the types of substance considered (over-the-counter preparations; prescription drugs; illegal substances), data collection method (e.g., extent to which the confidentiality of self-reports is guaranteed) and time period investigated (e.g., point vs. lifetime prevalence; Franke et al., 2014). Based on self-report questionnaires administered to large samples it has been reported that 5\% in the year 2010/2011 (Middendorff et al., 2012) and 6\% in 2014/2015 (Middendorff et al., 2015) of German university students abuse prescription drugs or illicit substances for "brain doping" during their years of study. Estimates of prevalence based on randomized response techniques that help to ensure the confidentiality of responses vary from $4 \%$ (Middendorff et al., 2015) to as much as $20 \%$ for 1 -year prevalence in a study including caffeine pills as a neuroenhancer (Dietz et al.,
2013). This latter figure is very similar to the reported abuse of prescription drugs for NE by research professionals (Maher, 2008). Maher reported that $20 \%$ of Nature readers from 60 nations who responded to an informal survey confessed to having used a prescription drug for NE at least once; however, Wiegel et al. (2015) reported that the lifetime prevalence of selfreported NE in a large sample of German university teachers and professors was only $1 \%$.

This study focused on university students' use of caffeine pills for NE. To date there has been only one pilot study of university students' use of coffee, caffeinated drinks, and caffeine pills in Germany (Franke et al., 2011). The authors concluded that 10.5\% of university students had used over-the-counter caffeine pills as neuroenhancers on at least one occasion.

\section{Psychological Factors Explaining NE in Students}

Several studies have analyzed students' motivation for NE. Eickenhorst et al. (2012) investigated German university students' motives for using prescription drugs and illegal substances as neuroenhancers. The main motives reported by their participants were a desire to enhance concentration and alertness or to increase cognitive functioning in general; to enable them to relax, cope with stress and withstand performance pressure and a fear of being disadvantaged if they did not use such substances. Middendorff et al. $(2012,2015)$ reported that when interviewed most students indicated that their main motive for NE was to maintain, rather than enhance performance and that use of prescription drugs and over-the-counter products was particularly frequent during exam preparations.

Some authors have tried to go beyond descriptions of users' motives and elucidate the psychological processes underlying NE behavior. Wolff and Brand (2013) regressed students' NE behavior (use of prescription drugs and over-the-counter products) on their underlying positive attitude to $\mathrm{NE}$ and Sattler and Wiegel (2013) reported that six-month prevalence of prescription drug NE was higher in students with high test anxiety.

Three studies investigated the influence of selected psychological factors on participants' decisions about trying NE using online factorial questionnaires (pseudoexperimental designs; see "Materials and Methods" section in this article). Sattler et al. (2013b) explored university students' and teachers' rationales for use of neuroenhancers using a hypothetical scenario in which they systematically varied several variables (indicated in italics): "A university [teacher/student] considers trying to enhance his cognitive performance for his [work/studies] by using a prescription drug which he does not require on medical grounds. He would be able to get the pills for free. A study that found that there is a 60 percent chance that the drug will improve cognitive performance by 250 percent caught his attention. The side effects were investigated: using the medication causes slight headaches in one out of 100,000 users. Possible additional side effects are unknown." Participants were asked to rate how willing they would be to use the substance described for NE if they were in the position of the person in 
the scenario. The authors controlled for several factors which they thought might influence decisions (e.g., moral evaluation of NE, conceptualized as the participant's internal norm). The authors, e.g., found that the probability and severity of sideeffects were negatively associated with willingness to use NE; internalization of social norms against use of neuroenhancers was also negatively associated with willingness to use NE. Sattler et al. (2013a) reported that NE was subject to a contagion effect: peer pressure (apparently high prevalence of substance use amongst peers) increased participants' willingness to use NE whereas formal prohibition and provision of information about health side-effects decreased willingness. Sattler et al. (2014) investigated drivers of and obstacles to drug use in university students from the perspective of economic decision theory, assuming that decisions about NE would be based on a rational evaluation of the probability that a substance would help users attain their goals. Two thirds of their participants staunchly refused to use a prescription drug for NE, a similar proportion to that found in the two previous studies (Sattler et al., 2013a,b). Low intrinsic motivation, high test anxiety and previous use of drugs for $\mathrm{NE}$ were associated with greater willingness to use NE in the future. As in earlier studies willingness to use neuroenhancers was negatively associated with high internalization of social norms against NE (i.e., moral disapproval of NE; see above) and positively associated with apparent peer prevalence.

\section{Call for Theory-Driven Analysis of NE Behavior}

Neuroenhancement is a multifaceted phenomenon (e.g., goals; factors governing decisions about use) that requires explanation at several levels (e.g., personal; social; environmental) based on a sound understanding of underlying psychosocial processes (e.g., evaluation of peer behavior). To date, however, most psychological research has been limited to identifying variables correlated with use of neuroenhancers and appears not to have been informed by psychological theory. Theory is an essential element of behavioral research; in particular the development of effective interventions is underpinned by explanatory models of behavior (Fishbein and Cappella, 2006). Experimental testing of theoretically derived hypotheses is superior to theoretically less-informed approaches, because it minimizes the risk of encountering arbitrary effects and overestimating the influence of variables (e.g., because of variable and case sampling errors).

This study aimed to address this research gap. It was based on the empirical findings from earlier psychological studies, but used an established theory of behavior to derive a model of the psychological determinants of NE behavior (cf. Zelli et al., 2015). Two other studies have used psychological theory to inform analyses of NE behavior. Wolff et al. (2014) used Job Demands Resources Theory to show that use of NE had a negative impact on university students' psychological perceptions of academic demands and interfered with their intrinsic motivation and Wolff et al. (2013) used the strength model of self-control to predict first use of NE. This study tested predictions derived from the Prototype Willingness Model (PWM; Gibbons et al., 1998). We investigated how information about peer behavior influenced associations between the psychological variables predicting university students' willingness and intention to use caffeine pills to enhance cognitive performance.

\section{The Prototype-Willingness Model}

At the core of the PWM (Gibbons et al., 1998) is the idea that sometimes persons will find themselves in situations which facilitate but do not compel particular behaviors. The model postulates that under such circumstance behavior is determined by the individual's willingness to perform the behavior in question rather than by a process of reflection with resulting plans and an intention at its end. Gibbons et al. (1998) defined willingness as an individual's openness to risk opportunity, i.e., to perform a risky behavior in the absence of a specific plan or intention to do so. Although willingness may be accompanied by a congruent intention this is not necessarily the case. According to the PWM willingness is a foundation from which overt behavior can emerge spontaneously and it can, at least temporarily, sustain that behavior (Gerrard et al., 2008).

The PWM is rooted in empirical analyses of adolescent risktaking behavior and developmental health psychology theory. The model suggests two pathways for behavioral regulation, the reasoned pathway, which culminates in an intention and the social reactive pathway, from which a degree of willingness emerges. The two pathways are connected in various ways, most importantly through a postulated predictive relationship between willingness and intention (but not vice versa). The PWM is illustrated in full in Figure 1.

The PWM assumes that future behavior is informed by past behavior. The variable 'past behavior' is a factor in all processes or steps of both the reasoned and social reactive pathways, which respectively, influence 'willingness' and 'intention' to perform a given behavior (Gibbons et al., 2009).

All variables in the reasoned pathway are drawn from the Theory of Reasoned Action (Fishbein and Ajzen, 1975, 2010) which treats 'attitude' as the sum of evaluations of a psychological object captured by evaluations of attribute dimensions such as good-bad, harmful-beneficial, pleasant-unpleasant and likeabledislikable (Ajzen, 2006). Another variable involved in the reasoned pathway is 'subjective norm,' which is defined as the perceived social pressure to engage or not engage in a given behavior. The PWM refers to descriptive norms (Gibbons et al., 1998). Items used to assess subjective norms should therefore capture individuals' perceptions of what significant others do (e.g., "Do you think your peers would use caffeine pills to enhance cognitive performance?") rather than what significant others think the respondent should do (injunctive norm; e.g., "Do you think that your peers would accept you taking caffeine pills for cognitive enhancement?"). 'Intention,' which is the PWM's most proximal predictor of behavior of the reasoned pathway, is based on a combination of attitude and descriptive norm.

The social reactive pathway is defined by certain variables' relationships with willingness. 'User prototype' is one of these variables. Gerrard et al. (2008) recommended that this variable should be measured using items such as "Take a moment to think about what kind of student in your age group uses caffeine pills to enhance cognitive functioning. We are not interested in anyone 


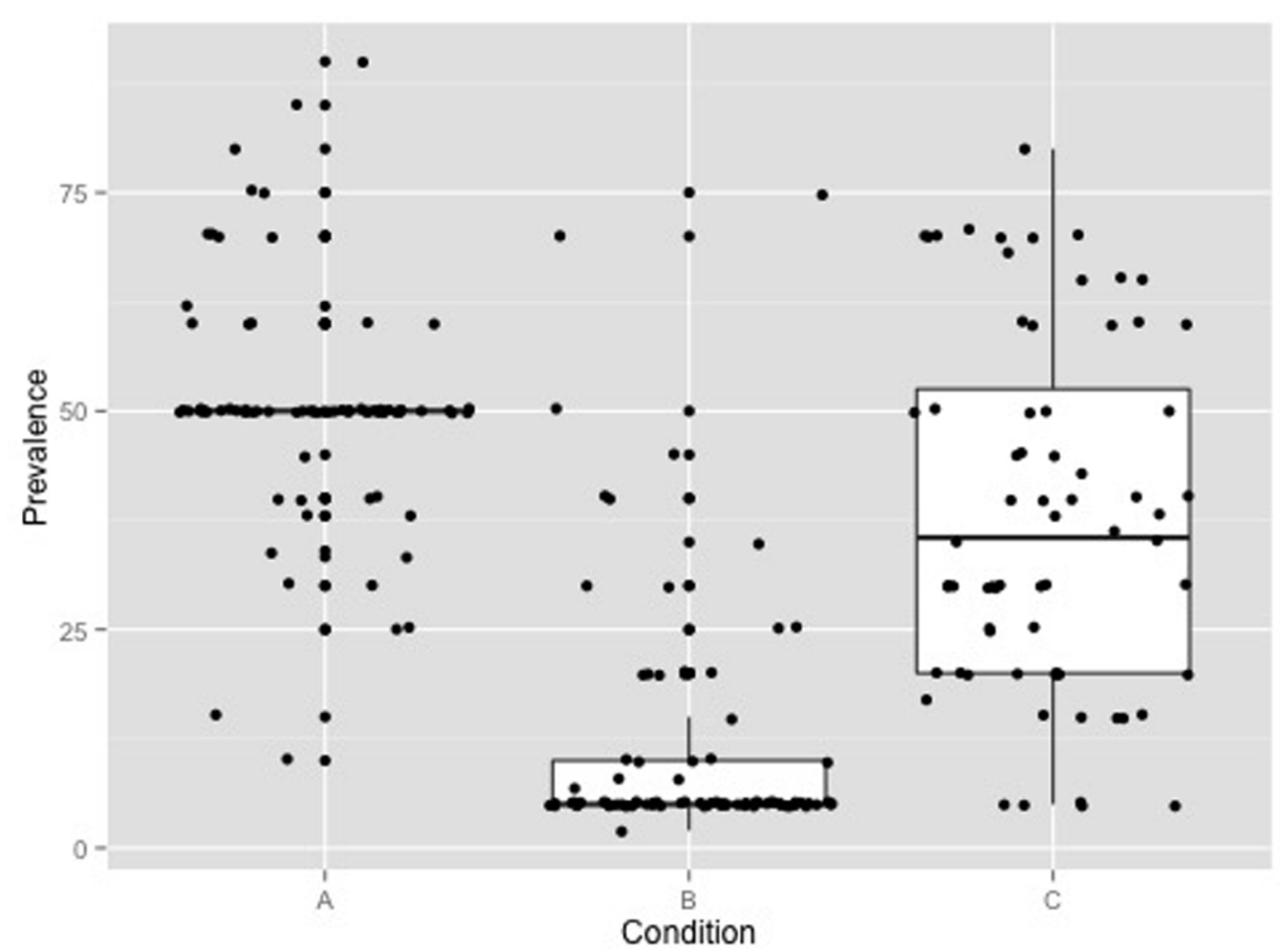

FIGURE 1 | Raw data and boxplots for participants' answers to the question in manipulation check 1 ("According to recent research what percentage of university students in Germany have used caffeine pills at least once to enhance their performance and improve their learning?") in experimental conditions A (50\% peer prevalence), B (5\%), and C (no information on peer prevalence).

in particular, just the typical person of your age who could do this. How [popular/smart/selfish] is this person?" In the context of the PWM a 'user prototype' is thus the sum of the characteristics of an imagined peer who engages in the target behavior (e.g., the 'typical' smoker). The social acceptability of a user prototype is positively associated with willingness to engage in the target behavior. 'Subjective norms' influence behavior via the reasoned pathway (through their association with 'intentions') and via the social reactive pathway, where they are postulated to be associated with 'willingness.' The socially reactive nature of the willingness construct is underscored by Gerrard et al.'s (2008) recommended items for measuring it, e.g., "Suppose you were studying with a group of friends and there were caffeine pills available. How willing would you be to take one?"

In the past few years, research on the PWM has accumulated in health psychology and a meta-analysis was published very recently (Todd et al., 2014). One of the conclusions from this meta-analysis was that the predictive power of the relationships postulated by the PWM is only marginally better in adolescent samples $\left(R^{2}=0.33\right)$ than in adult samples $\left(R^{2}=0.29\right)$, suggesting that although the model was developed to predict risk-taking behavior in adolescents it may be just as useful for predicting adult behavior. It is important to note that the meta-analysis did not test the full set of relationships involving PWM variables; it focused on the postulated correlations between prototypes, willingness and intentions and thus corroborated the postulated processes of the social reactive path. Most importantly the meta-analysis revealed that although willingness and intention are sometimes highly correlated they also act as independent predictors of risk behavior. Willingness explained an additional $4.9 \%$ of variance in behavior after intentions had been taken into account (Todd et al., 2014). The postulated correlations between variables in the reasoned pathway have been tested in several other PWM-related studies (e.g., the association between past behavior and attitude, Gibbons et al., 1998; pairwise associations between norms and intentions and willingness, Gerrard et al., 2008) and in meta-analyses which have confirmed the pattern of relationships among attitude, norms, intentions, and behavior (Armitage and Conner, 2001) postulated in the Theory of Planned Behavior (Ajzen, 1991).

Astonishingly, however, there is a dearth of empirical analyses of the full set of PWM variables using, e.g., multivariate model testing methodology.

\section{This Study}

Neuroenhancement research (e.g., Wolff and Brand, 2013; Schelle et al., 2014), research in related domains (e.g., doping in sport, Ntoumanis et al., 2014) and behavior change research more generally (e.g., McEachan et al., 2011) have shown that variables used in social cognitive theories, especially attitude, perceived social norms, and intentions (e.g., Fishbein and Ajzen, 1975, 2010), are important psychological predictors 
of behavior. Recently university students' willingness to use psychoactive substances for performance enhancement emerged as an empirically useful dependent variable in NE research (e.g., Sattler et al., 2013b). The PWM is a comprehensive psychological theory of behavior that integrates all these variables. The PWM postulates two interacting pathways of behavioral regulation: the reasoned action path, in which attitudes and norms predict intention (the most proximal predictor of behavior) and the social reactive path, which integrates information from prototypes, attitudes, and norms to form another proximal predictor of behavior, i.e., behavioral willingness. The PWM thus represents an integrative psychological framework for the study of NE behavior.

Earlier research indicated that $10 \%$ of German university students use caffeine-containing products with the purpose to enhance cognitive performance on at least one occasion during their student life (Franke et al., 2011). Use of drugs for NE carries health risks (Schermer et al., 2009) and students consider it a risky behavior (Sattler et al., 2014). Peer behavior and peer pressure have been shown to be factors in university students' decisions about use of enhancers (Sattler et al., 2013a).

In our investigation, we sought to manipulate groups of university students into believing either that use of caffeine pills for NE was rather widespread amongst their peer group (experimental condition A: prevalence given as $50 \%$; i.e., five times more than the actual estimated prevalence) or rare (condition B: prevalence given as 5\%; i.e., half the estimated prevalence). A third group received no information about prevalence and served as a control group (condition C). We investigated use of caffeine pills specifically. Caffeine is available in over-the-counter products and in higher doses as a prescription drug. We deliberately restricted our investigation to use of caffeine in pill form, because we assumed that such products were more likely to be seen as 'pharmaceuticals' than, for example, caffeinated energy drinks which might be perceived as an everyday consumable.

First of all, we tested whether the PWM could be used to describe university students' willingness and intentions with respect to use of NE. Our second research question was based on the assumption that university students would have some sort of subjective perception of the prevalence of NE in their peer group and that this perception would shape their thinking about NE. We explored whether experimentally manipulating information about the prevalence of use of caffeine pills for NE amongst peers (high prevalence; low prevalence; no prevalence information) would influence the behavioral determinants specified in the PWM (attitude, subjective norm, user prototype, willingness, and intention) and the associations between these variables and willingness or intention to engage in the target behavior.

\section{MATERIALS AND METHODS}

This study was conducted online as a factorial survey experiment (Jasso, 2006). This method enables the researcher to determine the influence of experimentally manipulated information on respondents' self-reported thoughts, feelings, and decisions. The
Questback EFS 10.6 software was used to run the experiment. The questionnaire was presented in German (i.e., all example items below are English translations from the German originals). The access link was distributed via Facebook and email thus creating a convenience sample drawn from the target population, university students in Germany. Data collection started in March 2015 and ended 3 weeks later in April 2015. The study was carried out in accordance with the recommendations of the ethical committee of the University of Potsdam (February 2015). All participants gave written, informed consent in accordance with the Declaration of Helsinki.

\section{Participants}

Four hundred and thirty-six university students clicked on the link giving access to our survey. Of these 386 (88.5\%) went beyond the title page and 305 (69.9\%) completed the questionnaire. Forty-nine students did not consent to having their data saved and analyzed on the last page, so the responses of 256 participants (58.7\% of those who clicked on the link) were stored for analysis. Twenty-five participants had provided incomplete data ( $>40 \%$ missing responses) and were therefore excluded from the final analyses, so the final sample consisted of data from 231 German-speaking university students of whom $75.8 \%$ were women $(M$ age $=23.5$ years \pm 2.7 ; range: $18-$ 35 years $)$ and $23.8 \%$ were men $(M$ age $=25.4$ years \pm 3.8 ; range: $20-40$ years). One 24 -year-old participant did not provide information about gender.

\section{Experimental Manipulation}

When they clicked through to the second page of the online questionnaire participants were randomly assigned to one of the three experimental conditions. Participants in condition A (fivefold overestimation of prevalence; Franke et al., 2011) read the following, "A few months ago a representative study showed that more than $50 \%$ of all university students in Germany use caffeine pills in order to enhance cognitive functioning and improve learning." Participants in condition B (i.e., 50\% underestimation of prevalence) read that only $5 \%$ of students used caffeine pills for NE. Participants in condition C read, "A few months ago a representative study showed that some university students in Germany use caffeine pills to enhance cognitive functioning and improve learning."

\section{Measures and Information}

\section{Past Behavior}

This indicator was intended to capture participants' historical NE behavior. They were asked four yes-no questions: "Have you ever used caffeine pills to enhance your cognitive performance and improve your learning?", "Have you ever used a caffeinated synthetic drink ('energy drink') to enhance your cognitive performance and improve your learning?," "Have you ever used any other synthetic substance to enhance your cognitive performance and improve your learning?", and "Have you ever drunk a cup of strong coffee or tea to enhance your cognitive performance and improve your learning?" Responses to these four questions were analyzed separately to characterize the sample, but the mean score was used in the main statistical 
analyses. McDonald's $\omega_{\mathrm{h}}=0.52$ that takes into account the dichotomous nature of these heterogeneous set of items indicated adequate general factor saturation.

\section{Subjective Norm}

The descriptive facet of this PWM variable was captured using three statements (see Hammer and Vogel, 2013) to which participants responded using a six-point, i.e., forced choice Likert-type scale ( $1=$ "totally disagree" to $6=$ "totally agree"): "Peers whose opinions I value use caffeine pills to enhance their cognitive performance and improve their learning," "People who are important to me would use caffeine pills to enhance their cognitive performance and learning if they were in my position," and "People around me have used caffeine pills to enhance their cognitive performance and improve their learning." The mean score for all three statements was used in the statistical analyses. In our sample the internal consistency of this scale was $\alpha=0.81$.

\section{Attitude}

Participants used a seven-point scale to rate use of caffeine pills to enhance cognitive performance and improve learning on five semantic differentials: 'bad-good,' 'unhealthy-healthy', 'rightwrong,' 'risky-safe,' and 'useless-useful' (see Ajzen, 2006). Higher values represented a more positive attitude toward use of caffeine pills for NE. The mean score from these five items was used for statistical analyses. In our sample the internal consistency of the scale was $\alpha=0.80$.

\section{User Prototype}

Participants were asked to imagine what a typical user of caffeine pills - perhaps a fellow student from their university - might be like (see Gibbons et al., 1998) and use a seven-point scale to describe that person in terms of five semantic differentials 'stupid-smart,' 'unpopular-well-liked,' 'motivated-unmotivated,' 'effective-ineffective,' and 'wrong-right.' The mean score for the scale was used in statistical analyses; higher means indicated greater social acceptability. In our sample the internal consistency of the scale was $\alpha=0.75$.

\section{Willingness}

Participants were asked to read two hypothetical scenarios and rate their willingness to use NE in each (see Gibbons et al., 1998). In scenario one, they read: "Suppose that a friend from your study program has written on Facebook that he recently used caffeine pills from the drugstore to improve his learning." Participants then rated how likely it was that they would take caffeine pills at some point using a six-point, i.e., forced choice Likert-type scale ( $1=$ "very unlikely" to $6=$ "very likely"). In scenario two, they read: "Usually you are well prepared for exams, but this time you feel tired and worn out. Although you are trying hard, you just don't seem able to prepare well for the forthcoming exam. You have the option of taking caffeine pills to dispel your fatigue and thus revise more effectively." Participants then responded to two questions ("If you had the opportunity, would you be willing to use caffeine pills?" and "Would you be willing to experiment with caffeine pills under some other circumstance if you had the opportunity to do so?") using a six-point, i.e., forced choice Likert-type scale $(1=$ "absolutely not willing" to $6=$ "perfectly willing." A mean score was calculated and used for statistical analyses, with higher scores indicating greater willingness to use caffeine. In our sample the internal consistency of this scale was $\alpha=0.88$.

\section{Intention}

This variable was measured very simply, by asking participants the single specific question "Do you intend to use caffeine pills for cognitive enhancement and to improve learning in preparation for your next exams?" Responses were given on a six-point, i.e., forced choice Likert-type scale $(1=$ "definitely not" to $6=$ "yes, definitely").

\section{Personal Details}

Participants were asked to provide their age and gender, and state whether they were currently enrolled as a university student.

\section{Manipulation Checks}

After responding to all the scales described above and providing personal data participants were asked "According to recent research what percentage of university students in Germany have used caffeine pills at least once to enhance their performance and improve their learning?" The response was entered in a free-text input field. Participants were also asked whether they thought there was anything suspicious, wrong, or strange about the questionnaire, and in particular if they thought they had been manipulated by the ways in which we gave or asked for information earlier in the questionnaire.

\section{Debriefing}

The last page of the questionnaire provided full information about the goals and procedure for the study. This included a statement of the actual estimated prevalence of use of caffeine pills for NE among university students (i.e., 10\%) and an explicit admission that we had tried to deceive our participants about this statistic in two experimental conditions. Participants had to tick response boxes to indicate that they had read and understood this information and consented to the confidential storage and analysis of their data for scientific use by the Division of Sport and Exercise Psychology of the University of Potsdam.

\section{Statistical Analyses}

SPSS 22.0 was used to calculate all descriptive statistics and for tests for group differences (ANOVA, MANOVA) and frequency distribution tests ( $\chi^{2}$ test). Structural equation modeling (SEM) with Amos 22.0 was used to determine whether our data were consistent with the PWM. General model fit was evaluated according to established criteria $(R M S E A<0.08, C F I \geq 0.95$, $S R M R<0.08$; e.g., $\mathrm{Hu}$ and Bentler, 1999; Beauducel and Wittmann, 2005) and a Bollen-Stine bootstrap (1000 iterations) was used to estimate confidence intervals for regression weights. A SEM multigroup moderation approach (Byrne, 2010) was used to investigate experimentally induced alterations in the relative predictive power of PWM predictors of willingness and intention (Lowry and Gaskin, 2014). These tests were carried out with a program created by Gaskin (2012) which calculates $z$-scores based on critical ratio tests of the multigroup model and unstandardized estimates. 
The significance level was set at $p<0.05$ for all analyses. Information from the second question of the manipulation check was evaluated qualitatively.

\section{RESULTS}

\section{Randomization Checks}

One-way ANOVA revealed no significant age differences between the three groups $[F(2)<1]$, indicating that in this respect the randomization procedure was successful. However, assessment of the gender ratios in the three groups indicated that there were fewer men $(n=12)$ and more women $(n=73)$ than expected in experimental condition $\mathrm{B}, \chi^{2}(2)=7.82, p<0.05$. Previous research suggests that male and female students may differ in their use of caffeine for NE (Franke et al., 2011), so this randomization error might have resulted in group differences in 'past behavior'; however, in practice there were no significant differences between conditions with regard to either historical use of specific substances (all ps n.s.; see below) or mean scores used in the statistical analyses (MANOVA results; see below). We therefore concluded that in spite of the randomization error (under-representation of men in condition B) the randomization process was successful overall.

\section{Manipulation Check}

The group mean responses to the question about what percentage of university students in Germany used caffeine pills for NE on at least one occasion were as follows: group A (fivefold overestimate of prevalence, i.e., $50 \%) M=50.39 \%(S D=13.24)$, group B (50\% underestimate of prevalence, i.e., $5 \%$ ) $M=11.28 \%$ $(S D=13.88)$ and group $\mathrm{C}$ (no information about prevalence) $M=38.18 \%(S D=20.34)$. This finding is illustrated in Figure 1 . There were group differences in responses [univariate ANOVA, $F(2,228)=130.2, p<0.01]$. The participants' free responses to the second manipulation check question indicated that participants did not see through the experimental manipulation.

\section{Description of Past Behavior}

Thirty-one participants (13.4\%) reported having used caffeine pills to enhance their performance on at least one occasion. Highdose synthetic caffeinated drinks ('energy drinks') had been used by $39.0 \%(n=90)$ and $14.3 \%(n=33)$ reported having used some other synthetic substance for NE, whilst $77.1 \%(n=178)$ had used a cup of strong coffee or tea, or some other natural substance for NE.

\section{Main Analyses PWM Model Fit}

Descriptive statistics for all variables are summarized in Table $\mathbf{1}$. The value of Mardia's multivariate kurtosis statistic was 1.24, indicating the multivariate normality of our data. The low variance inflation factor of 1.7 suggested that multicollinearity could be dismissed as a possible source of bias.

Structural equation modeling indicated that our data were a good fit with the predictions of the PWM after we had taken into
TABLE 1 | Descriptive statistics for all variables included in SEM.

\begin{tabular}{lllcc}
\hline Variables & $\boldsymbol{M}(\boldsymbol{S D})$ & Range & Skewness & Kurtosis \\
\hline Past behavior & $1.36(0.26)$ & $1-2$ & 0.68 & 0.12 \\
Attitude & $2.88(1.04)$ & $1-5.80$ & 0.06 & -0.23 \\
Subjective norm & $2.25(1.19)$ & $1-6$ & 0.79 & -0.47 \\
User prototype & $4.34(0.90)$ & $1-7$ & -0.09 & 0.40 \\
Intention & $2.13(1.38)$ & $1-6$ & 1.04 & 0.08 \\
Willingness & $3.00(1.39)$ & $1-6$ & 0.21 & -1.08 \\
\hline
\end{tabular}

$N=231$ for all variables.

account additional correlations between 'past behavior' and the residual variance in 'intention' $(r=0.18, p<0.05)$ and between 'past behavior' and the residual variance in 'willingness' $(r=0.30$, $p<0.01), \chi^{2}(1)=1.95, p=0.16$; RMSEA $=0.06$; CFI $=0.99$, $S R M R=0.01$. The structural model with regression weights for relationships between variables (bootstrapped CIs in Table 2) and determination coefficients for variables is displayed in Figure 2A.

Regression weights for the association between 'attitude' and 'intention' in the reasoned action pathway and the sequence of associations linking 'past behavior' to 'user prototype' to 'willingness' in the social reaction path were all low (all $\left.\beta_{\text {stand. }} \leq 0.10\right)$. 'Willingness' had a substantial effect on 'intention' $\left(\beta_{\text {stand. }}=0.62\right)$. The model explained $38 \%$ of variance in 'willingness' and $61 \%$ of variance in 'intention.'

In summary, our main hypothesis, that the PWM can be applied to university students' willingness and intentions with respect to use of caffeine pills for NE, was supported.

\section{Experimental Effects on Variable Means}

The MANOVA omnibus test revealed no significant effect of experimental condition on the six PWM variables, $F(12,448)=0.91$, n.s. We therefore concluded that provision of experimentally manipulated information about peer behavior did not produce group mean differences in any of the investigated variables.

\section{Experimental Effects on Regression Paths}

Model fit indices remained acceptable for the multigroup estimation model, $\chi^{2}(3)=6.85, p=0.08 ; R M S E A=0.07$; $C F I=0.99, S R M R=0.01$. Provision of experimentally manipulated evidence about prevalence lowered regression

TABLE 2 | Bootstrapped 95\% Cls for regression weight ( $\left.\beta_{\text {stand. }}\right)$.

\begin{tabular}{llllc}
\hline Parameters & & & Lower boundary & Upper boundary \\
\hline Past behavior & $\rightarrow$ & Subjective norm & 0.37 & 0.58 \\
Past behavior & $\rightarrow$ & User prototype & -0.08 & 0.19 \\
Past behavior & $\rightarrow$ & Attitude & 0.34 & 0.53 \\
Subjective norm & $\rightarrow$ & Willingness & 0.08 & 0.30 \\
Attitude & $\rightarrow$ & Willingness & 0.28 & 0.50 \\
User prototype & $\rightarrow$ & Willingness & -0.06 & 0.14 \\
Subjective norm & $\rightarrow$ & Intention & 0.01 & 0.24 \\
Attitude & $\rightarrow$ & Intention & -0.01 & 0.21 \\
Willingness & $\rightarrow$ & Intention & 0.51 & 0.72 \\
\hline
\end{tabular}

$N=231$ for all variables. 

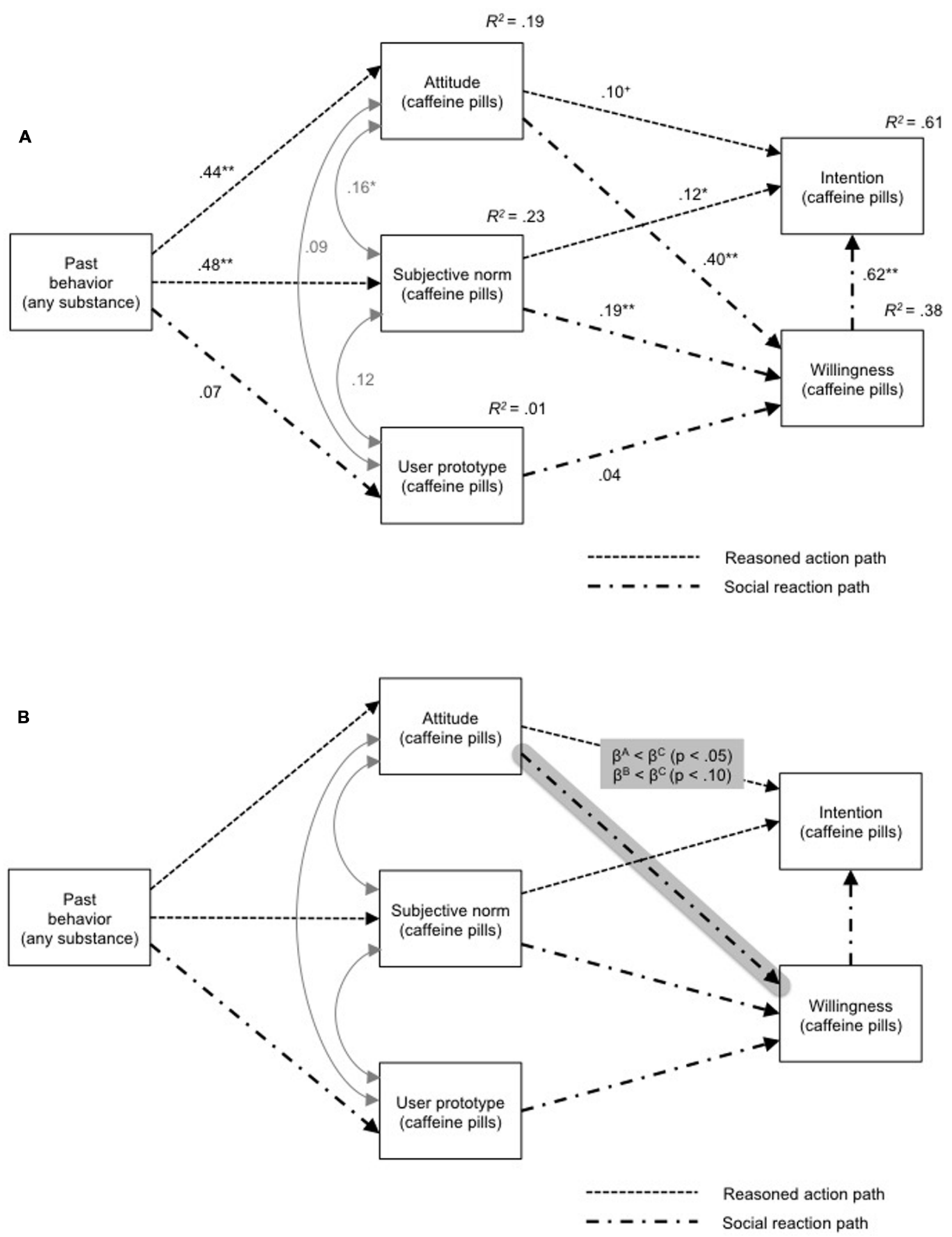

FIGURE 2 | The Prototype Willingness Model (Gibbons et al., 2009) with results from SEM in (A) ( $R^{2}$ for PWM variables and $\beta_{\text {stand. }}$ for paths) and the illustration of experimental effects (B).

weight for the associations between 'attitude' and 'willingness' in condition A by $45 \%$ relative to the control group, which did not receive any prevalence information; a similar effect (lowered regression weight by $40 \%$ ) appeared as a statistical trend in condition B (Figure 2B; condition A: $\beta_{\text {stand. }}=0.31, p<0.01$; condition B: $\beta_{\text {stand. }}=0.34, p<0.01$; condition C: $\beta_{\text {stand. }}=0.57$, 
$p<0.01)$. This means that in our sample provision of information about prevalence of use of caffeine pills for NE decreased the predictive value of attitude to use of caffeine for $\mathrm{NE}$ on willingness to do so.

\section{DISCUSSION}

Testing predictions derived from a theory of behavior minimizes the risk of reporting random effects and overestimating relationships; it is thus a suitable strategy for uncovering the psychological mechanisms underlying behavior and behavior change (Zelli et al., 2015). This study showed that the PWM, an established social cognitive theory of behavior, can be used to describe university students' willingness and intention to use caffeine pills to enhance their academic performance (NE). On the basis of this result and previous findings on the effects of peer pressure (Sattler et al., 2013a,b, 2014) we explored whether providing information about the prevalence of a behavior amongst peers - in this case use of caffeine pills for NE - would influence motivational variables and their interrelationships. We found that attitude was a less powerful predictor of willingness to use caffeine pills in experimental condition $\mathrm{A}$, in which participants were informed that recent research indicated that $50 \%$ of all university students in Germany used caffeine for NE. There was a trend in the same direction $(p<0.10)$ in condition $B$, where participants were informed that only $5 \%$ of peers used caffeine for NE.

Interestingly, in this investigation attitude was a much weaker predictor of intention than in other investigations of problematic consumption behavior (e.g., $r_{+}=0.62$ in a meta-analysis of intention to consume alcohol, Cooke et al., 2014; $r=0.55$ in a meta-analysis of athletes' intention to use doping substances, Ntoumanis et al., 2014). The most likely explanation for this is that we chose to measure a very specific intention, i.e., intention to use caffeine, in pill form, in the next exam period. The relatively skewed distribution $(z=1.04)$ of rather low intentions $(M=2.13 \pm 1.38$ on a six-point Likert scale) corroborates this explanation. Our participants' willingness to use caffeine for NE was somewhat higher $(M=3.00 \pm 1.39)$ and barely skewed $(z=0.21)$. We consider that our very straightforward way of measuring a very specific intention represents a valid, reliable indication of intention with respect to the behavior in question, we therefore argue that the 'willingness' construct is the more interesting proximal psychological predictor of NE (Sattler et al., 2014) and should be investigated further. In particular, we recommend research into the conceptual relationship between willingness and intention (empirical data from many studies based on the PWM suggest that the two constructs are fairly highly correlated, c.f. Todd et al., 2014; $\beta_{\text {stand. }}=0.62$ in our sample). We think that willingness to engage in a given behavior may be much more sensitive to changes in motivational predictors (e.g., in attitude) and to situational factors than an intention to do so. A fundamental assumption of the PWM is that willingness is the basis of socially reactive, i.e., 'spontaneous' or unplanned behavior; this suggests that willingness would be a suitable target for public health interventions.

Structural equation modeling modeling enabled us to estimate correlations between the PWM variable past behavior and the residual variance in willingness and intention with respect to the relevant behavior. In accordance with Wolff and Brand's (2013) behavioral approach to investigating motivation to use NE we measured our participants' past NE behavior without regard for the substances involved although we measured willingness and intention in relation to a specific substance, namely caffeine pills. Our data suggests that an individual's general disposition to use neuroenhancers is a product of unobserved variables in addition to the PWM variables we measured. These unidentified variables have a considerable impact on willingness and intention to use specific neuroenhancers and perhaps NE more generally. Candidates for these thus far unidentified variables might be found in the psychological roots of NE behavior and perhaps in the goals at which NE is directed. More generally we endorse Lazuras's (2015) recommendation, made in relation to research on doping in sport (see Zelli et al., 2015, for parallels between doping and NE), that researchers should develop coherent explanatory models that account for environmental influences, demographic variables, culture, and exposure to information in the media.

The PWM variable past behavior was linked to social norm and attitude but had almost no relationship with user prototype. Participants' images of the "typical user" (Gibbons et al., 2009) of caffeine pills, including social acceptability (i.e., user prototype) also had little impact on willingness to use NE. In the terminology of the PWM the basis for socially reactive use of caffeine pills to improve academic performance seems to be the strong influence of attitude on willingness; user prototype appears to play little role in socially reactive $\mathrm{NE}$ in this instance. This may be due to our choice of example neuroenhancer, namely caffeine pills. Caffeine pills may be more likely than other caffeine products such as 'energy drinks' to be treated as a pharmaceutical that should not be taken recklessly, or in response to peer pressure. If we assume that university students are better educated than the general population we might expect them to be more resistant to social influences and generally less prone to unplanned, socially reactive behavior and hence that user prototype would be a stronger predictor of NE behavior in other samples and in relation to other substances.

The experimental manipulation of information about prevalence of use of caffeine for NE amongst peers (rather widespread; rare) did not affect participants' subjective norm for this behavior, in other words quantitative information about prevalence did not appear to be internalized and incorporated into belief systems immediately. This may be because the information given in conditions A (50\% prevalence, i.e., a fivefold overstatement of actual estimated prevalence) and $B$ (5\%, i.e., half the estimated prevalence) did not correspond with our participants' existing perceptions based on personal observations. The responses of the university students in condition $\mathrm{C}$ (no information on peer prevalence) to the manipulation check question about prevalence (see Figure 1) 
provide some indication of students' pre-existing perceptions of $\mathrm{NE}$ prevalence and suggest that such perceptions vary widely. We suggest that receiving new information about peer prevalence might weaken the association between attitude to a given behavior and willingness to engage in it. Our finding that in condition $\mathrm{A}$ the predictive power of attitude with respect to willingness decreased by $45 \%$ might be taken as andication that our participants had begun to reflect on their point of view in response to the rather surprising - i.e., inaccurate, experimentally manipulated - information about the prevalence of NE amongst their peer group. This finding reinforces our main contention, shared by other authors (Wolff and Brand, 2013; Wolff et al., 2014; Zelli et al., 2015), which is that social cognitive theories which define relationships between, e.g., attitude and other psychological predictors of behavior (in this case NE) provide valuable insight into the psychological mechanisms underlying behavior change and hence can be used to develop behavior prevention programs.

The limitations of this research should be acknowledged. First of all, psychological theories such as the PWM are intended to predict behavior. We have neither predicted a temporal relationship (e.g., that past behavior influences attitude) nor measured observed behavior (e.g., use of caffeine pills in the next exam period) following an experimental treatment. We experimentally manipulated one variable (information about prevalence) and were thus able to make causal inferences related to this manipulation (providing information about prevalence reduced the influence of attitude to $\mathrm{NE}$ on willingness to engage in it). We are, however, unable to draw conclusions about the validity of theoretical assumptions about the causal relationships between other variables (e.g.,

\section{REFERENCES}

Ajzen, I. (1991). The theory of planned behavior. Organ. Behav. Hum. 50, 179-211. doi: 10.1016/0749-5978(91)90020-T

Ajzen, I. (2006). Constructing a Theory of Planned Behavior Questionnaire. Available at: http://people.umass.edu/aizen/tpb.html

Armitage, C., and Conner, M. (2001). Efficacy of the theory of planned behaviour: a meta-analytic review. Br. J. Soc. Psychol. 40, 471-499. doi: $10.1348 / 014466601164939$

Beauducel, A., and Wittmann, W. W. (2005). Simulation study on fit indexes in CFA based on data with slightly distorted simple structure. Struct. Equ. Model. 12, 41-75. doi: 10.1207/s15328007sem1201_3

Byrne, B. M. (2010). Structural Equation Modeling with Amos: Basic Concepts, Applications, and Programming, 2nd Edn. New York, NY: Taylor and Francis.

Clark, V. P., and Parasuraman, R. (2014). Neuroenhancement: enhancing brain and mind in health and in disease. Neuroimage 85, 889-894. doi: 10.1016/j.neuroimage.2013.08.071

Cooke, R., Dahdah, M., Norman, P., and French, D. P. (2014). How well does the theory of planned behaviour predict alcohol consumption? A systematic review and meta-analysis. Health Psychol. Rev. 17, 1-20. doi: 10.1080/17437199.2014.947547

de Berker, A. O., Bikson, M., and Bestmann, S. (2013). Predicting the behavioral impact of transcranial direct current stimulation: issues and limitations. Front. Hum. Neurosci. 7:613. doi: 10.3389/fnhum.2013.00613

Dietz, P., Striegel, H., Franke, A. G., Lieb, K., Simon, P., and Ulrich, R. (2013). Randomized response estimates for the 12-month prevalence of cognitiveenhancing drug use in university students. Pharmacotherapy 33, 44-50. doi: 10.1002/phar.1166 the direction of the association between subjective norms and willingness; Figure 1) as our evidence on this was correlational. Longitudinal studies are needed to draw conclusions about the consequences of changes in motivational determinants. Another limitation of our study is that our analyses were based on data from an ad hoc sample of university students which may not have been representative of the population. Although, we are optimistic that our findings are valid further studies are needed to corroborate our findings and interpretation.

\section{CONCLUSION}

We hope that future research will be theoretically informed, seeking to address research questions derived from and relevant to psychological theory. By taking this kind of approach we have shown that information about the prevalence of a behavior amongst peers - in this case use of NE to improve academic performance - might have a deterrent effect via attitude to NE and willingness to engage in NE. The approach described in this study might be particularly useful for the designers of public health campaigns.

\section{AUTHOR CONTRIBUTIONS}

$\mathrm{RB}$ developed this research question. HK conducted the empirical part of the study as a part of her bachelor thesis. RB and $\mathrm{HK}$ jointly re-analyzed the data, adjusted and broadened the chain of arguments, and then cooperatively wrote this report.

Eickenhorst, P., Vitzthum, K., Klapp, B. F., Groneberg, D., and Mache, S. (2012). Neuroenhancement among german university students: motives, expectations, and relationship with psychoactive lifestyle drugs. J. Psychoactive Drugs 44, 418-427. doi: 10.1080/02791072.2012.736845

Farah, M. J., Smith, M. E., Ilieva, I., and Hamilton, R. H. (2014). Cognitive enhancement. WIREs Cogn. Sci. 5, 95-103. doi: 10.1002/wcs. 1250

Fishbein, M., and Ajzen, I. (1975). Belief, Attitude, Intention, and Behavior: An Introduction to Theory and Research. Reading, MA: Addison-Wesley.

Fishbein, M., and Ajzen, I. (2010). Predicting and Changing Behavior: The Reasoned Action Approach. New York, NY: Psychology Press.

Fishbein, M., and Cappella, J. N. (2006). The role of theory in developing effective health communications. J. Commun. 56, S1-S17. doi: 10.1111/j.14602466.2006.00280.x

Franke, A. G., Bagusat, C., Rust, S., Engel, A., and Lieb, K. (2014). Substances used and prevalence rates of pharmacological cognitive enhancement among healthy subjects. Eur. Arch. Psychiatry Clin. Neurosci. 264(Suppl. 1), S83-S90. doi: 10.1007/s00406-014-0537-1

Franke, A. G., Christmann, M., Bonertz, C., Fellgiebel, A., Huss, M., and Lieb, K. (2011). Use of coffee, caffeinated drinks and caffeine tablets for cognitive enhancement in pupils and students in Germany. Pharmacopsychiatry 44, 331-338. doi: 10.1055/s-0031-1286347

Franke, A. G., and Lieb, K. (2010). Pharmakologisches neuroenhancement und "Hirndoping": chancen und Risiken [Pharmacological neuroenhancement and brain doping: Chances and risks]. Bundesgesundheitsblatt Gesundheitsforschung Gesundheitsschutz 53, 853-859. doi: 10.1007/s00103-0101105-0

Gaskin, J. (2012). Group Differences, Stats Tools Package. Available at: http:// statwiki.kolobkreations.com 
Gerrard, M., Gibbons, F. X., Houlihan, A. E., Stock, M. L., and Pomery, E. A. (2008). A dual-process approach to health risk decision making: the prototype willingness model. Dev. Rev. 28, 29-61. doi: 10.1016/j.dr.2007.10.001

Gibbons, F. X., Gerrard, M., Blanton, H., and Russell, D. W. (1998). Reasoned action and social reaction: willingness and intention as independent predictors of health risk. J. Pers. Soc. Psychol. 74, 1164-1180. doi: 10.1037/00223514.74.5.1164

Gibbons, F. X., Houlihan, A. E., and Gerrard, M. (2009). Reason and reaction: the utility of a dual-focus, dual-processing perspective on promotion and prevention of adolescent health risk behaviour. Br. J. Health Psychol. 14(Pt 2), 231-248. doi: 10.1348/135910708X376640

Hammer, J. H., and Vogel, D. L. (2013). Assessing the utility of the willingness/prototype model in predicting help-seeking decisions. J. Couns. Psychol. 60, 83-97. doi: 10.1037/a0030449

Hildt, E., and Franke, A. G. (2013). Cognitive Enhancement: An Interdisciplinary Perspective. New York, NY: Springer.

Hu, L. T., and Bentler, P. M. (1999). Cutoff criteria for fit indexes in covariance structure analysis: Conventional criteria versus new alternatives. Struct. Equ. Model. 6, 1-55. doi: 10.1080/10705519909540118

Ilieva, I., Boland, J., and Farah, M. J. (2013). Objective and subjective cognitive enhancing effects of mixed amphetamine salts in healthy people. Neuropharmacology 64, 496-505. doi: 10.1016/j.neuropharm.2012.07.021

Ishak, W. W., Ugochukwu, C., Bagot, K., Khalili, D., and Zaky, C. (2012). Psychological effects and impact on well-being and quality of life - a literature review. Innov. Clin. Neurosci. 9, 25-34.

Jasso, G. (2006). Factorial survey methods for studying beliefs and judgments. Soc. Methods Res. 34, 334-423. doi: 10.1177/0049124105283121

Jongh, R., Bolt, I., Schermer, M., and Olivier, B. (2008). Botox for the brain: enhancement of cognition, mood and pro-social behavior and blunting of unwanted memories. Neurosci. Behav. Rev. 32, 760-776. doi: 10.1016/j.neubiorev.2007.12.001

Kipke, R. (2013). "What is cognitive enhancement and is it justified to point out this kind of enhancement within the ethical discussion?," in Cognitive Enhancement: An Interdisciplinary Approach, eds E. Hildt and A. G. Franke (Berlin: Springer), $145-157$.

Lazuras, L. (2015). "Social-cognitive predictors of doping use: an integrative approach," in The Psychology of Doping, eds V. Barkoukis, L. Lazuras, and H. Tsorbatzoudis (London: Routledge), 44-61.

Lowry, P. B., and Gaskin, J. (2014). Partial least squares (PLS) structural equation modeling (SEM) for building and testing behavioral causal theory: when to choose it and how to use it. IEEE T Prof Commun 57, 123-146. doi: 10.1109/TPC.2014.2312452

Maher, B. (2008). Poll results: look who's doping. Nature 452, 674-675. doi: $10.1038 / 452674 \mathrm{a}$

Maier, L. J., and Schaub, M. P. (2015). The use of prescription drugs and drugs of abuse for neuroenhancement in Europe: not widespread but a reality. Eur. Psychol. 20, 155-166. doi: 10.1027/1016-9040/a000228

McEachan, R. R. C., Conner, M., Taylor, N. J., and Lawton, R. J. (2011). Prospective prediction of health-related behaviours with the theory of planned behaviour: a meta-analysis. Health Psychol. Rev. 5, 97-144. doi: 10.1080/17437199.2010.521684

Middendorff, E., Poskowsky, J., and Becker, K. (2015). Formen der Stresskompensation und Leistungssteigerung bei Studierenden: Wiederholungsbefragung des HISBUS-Panels zu Verbreitung und Mustern studienbezogenen Substanzkonsums. Hannover: DZHW.

Middendorff, E., Poskowsky, J., and Isserstedt, W. (2012). Formen der Stresskompensation und Leistungssteigerung bei Studierenden: HISBUSBefragung zur Verbreitung und $z u$ Mustern von Hirndoping und Medikamentenmissbrauch. HIS: Forum Hochschule.

Mueller, C. P., and Schumann, G. (2011). Drugs as instruments: a new framework for non-addictive psychoactive drug use. Behav. Brain Sci. 34, 293-310. doi: $10.1017 / \mathrm{s} 0140525 \times 11000057$
Ntoumanis, N., Ng, J. Y., Barkoukis, V., and Backhouse, S. (2014). Personal and psychosocial predictors of doping use in physical activity settings: a metaanalysis. Sport Med. 44, 1603-1624. doi: 10.1007/s40279-014-0240-4

Partridge, B. J., Bell, S. K., Lucke, J. C., Yeates, S., and Hall, W. D. (2011). Smart drugs "as common as coffee": media hype about neuroenhancement. PLoS ONE 6:e28416. doi: 10.1371/journal.pone.0028416

Sattler, S., Forlini, C., Racine, E., and Sauer, C. (2013a). Impact of contextual factors and substance characteristics on perspectives toward cognitive enhancement. PLoS ONE 8:e71452. doi: 10.1371/journal.pone.0071452

Sattler, S., Sauer, C., Mehlkop, G., and Graeff, P. (2013b). The rationale for consuming cognitive enhancement drugs in university students and teachers. PLoS ONE 8:e68821. doi: 10.1371/journal.pone.0068821

Sattler, S., Mehlkop, G., Graeff, P., and Sauer, C. (2014). Evaluating the drivers of and obstacles to the willingness to use cognitive enhancement drugs: the influence of drug characteristics, social environment, and personal characteristics. Subst. Abuse Treat. Prev. Policy 9:8. doi: 10.1186/1747-597X-9-8

Sattler, S., and Wiegel, C. (2013). Cognitive test anxiety and cognitive enhancement: the influence of students' worries on their use of performance-enhancing drugs. Subst. Use Misuse 48, 220-232. doi: 10.3109/10826084.2012.751426

Schelle, K. J., Faulmüller, N., Caviola, L., and Hewstone, M. (2014). Attitudes toward pharmacological cognitive enhancement - a review. Front. Syst. Neurosci. doi: 10.3389/fnsys.2014.00053 [Epub ahead of print].

Schermer, M., Bolt, I., De Jongh, R., and Olivier, B. (2009). The future of psychopharmacological enhancements: expectations and policies. Neuroethics 2, 75-87. doi: 10.1007/s12152-009-9032-1

Todd, J., Kothe, E., Mullan, B., and Monds, L. (2014). Reasoned versus reactive prediction of behaviour: a meta-analysis of the prototype willingness model. Health Psychol. Rev. doi: 10.1080/17437199.2014.922895 [Epub ahead of print].

Wiegel, C., Sattler, S., Göritz, A. S., and Diewald, M. (2015). Work-related stress and cognitive enhancement among university teachers. Anxiety Stress Copin 29, 100-117. doi: 10.1080/10615806.2015.1025764

Wolff, W., Baumgarten, F., and Brand, R. (2013). Reduced self-control leads to disregard of an unfamiliar behavioral option: an experimental approach to the study of neuroenhancement. Subst. Abuse Treat. Prev. Policy 8:41. doi: 10.1186/1747-597X-8-41

Wolff, W., and Brand, R. (2013). Subjective stressors in school and their relation to neuroenhancement: a behavioral perspective on students' everyday life "doping". Subst. Abuse Treat. Prev. Policy 8:23. doi: 10.1186/1747-597X-8-23

Wolff, W., Brand, R., Baumgarten, F., Lösel, J., and Ziegler, M. (2014). Modeling students' instrumental (mis-)use of substances to enhance cognitive performance: neuroenhancement in the light of job-demands-resources theory. BioPsychoSoc. Med 8:12. doi: 10.1186/1751-0759-8-12

Zelli, A., Lucidi, F., and Mallia, L. (2015). The complexity of neuroenhancement and the adoption of a social cognitive perspective. Front. Psychol. 6:1880. doi: 10.3389/fpsyg.2015.01880

Zohny, H. (2015). The myth of cognitive enhancement drugs. Neuroethics 8, 257-269. doi: 10.1007/s12152-015-9232-9

Conflict of Interest Statement: The authors declare that the research was conducted in the absence of any commercial or financial relationships that could be construed as a potential conflict of interest.

The reviewer Christopher Fullerton and handling Editor declared their shared affiliation, and the handling Editor states that the process nevertheless met the standards of a fair and objective review.

Copyright (c) 2016 Brand and Koch. This is an open-access article distributed under the terms of the Creative Commons Attribution License (CC BY). The use, distribution or reproduction in other forums is permitted, provided the original author(s) or licensor are credited and that the original publication in this journal is cited, in accordance with accepted academic practice. No use, distribution or reproduction is permitted which does not comply with these terms. 